\title{
Skin inspired fractal strain sensors using a copper nanowire and graphite microflake hybrid conductive network $\dagger$
}

Cite this: DOI: $10.1039 / \mathrm{c} 6 \mathrm{nr} 04056 \mathrm{j}$

Received 19th May 2016, Accepted 21st August 2016

DOI: 10.1039/c6nr04056j

N. N. Jason, ${ }^{a}$ S. J. Wang ${ }^{b, c}$ and W. Cheng ${ }^{a}$ *

www.rsc.org/nanoscale

This work demonstrates a facile "paint-on" approach to fabricate highly stretchable and highly sensitive strain sensors by combining one-dimensional copper nanowire networks with two-dimensional graphite microflakes. This paint-on approach allows for the fabrication of electronic skin (e-skin) patches which can directly replicate with high fidelity the human skin surface they are on, regardless of the topological complexity. This leads to high accuracy for detecting biometric signals for applications in personalised wearable sensors. The copper nanowires contribute to high stretchability and the graphite flakes offer high sensitivity, and their hybrid coating offers the advantages of both. To understand the topological effects on the sensing performance, we utilized fractal shaped elastomeric substrates and systematically compared their stretchability and sensitivity. We could achieve a high stretchability of up to $600 \%$ and a maximum gauge factor of 3000 . Our simple yet efficient paint-on approach enabled facile fine-tuning of sensitivity/stretchability simply by adjusting ratios of $1 D$ vs. $2 D$ materials in the hybrid coating, and the topological structural designs. This capability leads to a wide range of biomedical sensors demonstrated here, including pulse sensors, prosthetic hands, and a wireless ankle motion sensor.

\section{Introduction}

We can think of the human skin as a pre-strained and therefore wrinkled elastic sheet. This facilitates biaxial strain by deforming in such a way that the majority of the strain is converted to compressional stress, thereby avoiding tears from forming on the skin due to excess longitudinal strain

${ }^{a}$ Department of Chemical Engineering, Faculty of Engineering, Monash University, Clayton 3800, Victoria, Australia

${ }^{b}$ International Tangible Interaction Design Lab (ITIDLab), Monash University, Clayton, Victoria 3800, Australia

${ }^{c}$ Interaction Design, Department of Design, Faculty of Art Design and Architecture, 900 Dandenong Road, Caulfield East 3145, Victoria, Australia

$\dagger$ Electronic supplementary information (ESI) available: Images S1-S8 and Videos S1-S3. See DOI: 10.1039/c6nr04056j concentration in isolated areas. In elastic sheets, on increasing the aspect ratio $(\alpha=L / W)$, where $L=$ length and $W=$ width of the sheet before strain, there is a transition where the majority of the forces experienced by the sheet turn to more of compressional stresses perpendicular to the width and less of longitudinal strains along the length, and vice versa. ${ }^{1}$ This allows a high $\alpha$ sheet to endure extremely large strains without tearing. It must be noted that the sheet is clamped at the two ends of the length of the sheet, with the clamp being at least as wide as the sheet itself. In essence, the human skin has a typical stretchability of less than $30 \%$, but it is still able to protect the body from injury by continuous monitoring of strain, pressure and temperature over the entire skin surface, especially the injury prone areas such as the joints. ${ }^{2}$

Much of the research work in this field was primarily based on simple patch based architectures with various nanomaterial based inks as the sensing element for strain detection. ${ }^{3-12}$ Examples include interlocking fibres, lateral un-entangling of carbon nanotubes for strain sensing, gold nanowire soaked tissue paper piezoresistive pressure sensors, etc. ${ }^{13-18}$ It has been demonstrated that multi-functionality in a single setup was possible by using rigid silicon in the form of thin serpentine shavings and they were connected to microelectronic components to fabricate a plethora of tattoo-like, skin attachable, integrated devices including self-similar or fractal interconnects, and strain, temperature, and electrostatic potential (EP) sensors. ${ }^{18-22}$ Complex functions such as cardio therapy have also been achieved using the same strategy. ${ }^{23}$ Building on this concept, a "cut-and-paste" e-skin has also recently been developed which actually doesn't utilize any nanomaterials at all, and instead uses commercially available metallic thin films. ${ }^{9,24}$ There have also been some novel 3-D printed architectures to fabricate e-skins. ${ }^{25}$

Despite the significant progress in e-skin sensors, the majority of them are made by fabrication of sensors first and attachment to the skin later. This strategy couldn't replicate the human skin surface textures with high fidelity, limiting their applications in personalised wearable electronics. Here, we describe a simple yet efficient 'paint-on' strategy which can 
1 circumvent this limitation, by using graphite microflakes (GrMFs) and copper nanowires (CuNWs) as the conducting/ sensing elements. Although CuNWs do tend to oxidise rapidly when exposed to air, this problem can be easily solved by using nickel based protective alloy shells on the NWs. ${ }^{12,26,27}$ The combination of GrMFs and CuNWs enables facile tuning of the desired sensitivity and stretchability. We could paint sensors directly on the human skin, fabric and other complex surfaces as required by the end user. As a result of being free from complex fabrication methods, the user has the freedom to manufacture a wide range of sensors with varying sensitivities and stretchabilities on virtually any substrate, regardless of the topological complexity. In addition to materialbased tuning, we could extend the stretchability by combining fractal design. ${ }^{21,22,28,29}$ With optimum design, we could achieve a high stretchability of up to $600 \%$ and a maximum gauge factor of 3000 . The wearable sensors on the skin can be easily washed away with water after use, and the smart sock can simply be removed like a normal sock and discarded as well. The total cost including the conductive paint, elastomeric substrates and sealants and the conductive thread per sensor is estimated to be less than AUD \$2. Moreover, the paintable sensors can be seamlessly integrated with wireless networks for continuous, real time biometric data collection, transmission and sharing.

To demonstrate the biomonitoring capabilities of the sensors the GrMF ink was directly painted onto the human skin and the resulting sensor was used to measure the ankle brachial pulse from the posterior tibial artery. A single sensor was also painted on the wrist and it could distinctly detect individual finger motions, palm flexion and extension. Finally, a group of three sensors using the GrMF/CuNW/GrMF ink was painted around the ankle, front and on both sides, to monitor the calcaneo-fibular, medial, and anterior-tibiofibular ligaments. The sensors were painted on a sock and coupled with a wireless platform to demonstrate a truly wearable and wirefree, untethered sensor package for sporting applications. This was used to collect real time ankle motion data simultaneously from all three sensors on a smart phone.

\section{Results and discussion}

The fabrication process for the sensors begins first with the synthesis of the CuNW and GrMF inks. The aqueous CuNW ink was made using the methodology specified in our previous work, ${ }^{9}$ and the GrMF ink was made by dissolving shavings of a commercial 9B water soluble graphite crayon in water. Both types of inks could be painted on elastomers. We thoroughly investigated their ink-on-elastomer behaviours with respect to applied strain. Three types of percolation networks formed here, namely, a CuNW based network made of one dimensional nanowire building blocks, a GrMF network made of two dimensional flakes arranged onto each other like scales on reptilian skin, and finally a hybrid network comprising the GrMF/CuNW/GrMF sandwich structure.
The NW based network has been thoroughly researched in previous reports. The deformation ability of the NWs dictates whether the change in resistance in a NW network comes from contact resistance, breaking of contact entirely between nanowires due to out of plane buckling and overstretching of the NW film. Moreover, if the NWs are bound to each other and the elastomer substrate quite rigidly then the resistance changes are due to the formation of cracks in the percolation network during strain. Fig. 1(A) shows the uniform network of CuNWs formed before the application of strain, but under the application of $50 \%$ strain we can see cracks in the NW film perpendicular to the direction of strain, as shown in Fig. 1(B) outlined in the red dashed box and also seen in Fig. S1. $\dagger$ The formation of compressional wrinkles parallel to the direction of strain is also clearly visible, Fig. $\mathrm{S} 1(\mathrm{a}-\mathrm{c})+\dagger$ This is a very clear example of the Poisson effect in play. Upon relaxation of the strain the cracks close up, but a seamless smooth film is not formed, Fig. 1(C). This illustrates that NW films are inherently prone to degradation due to their inability to return to their original positions post deformation, which is consistent with previous NW-based systems. ${ }^{8,30,31}$

The GrMFs form a percolation network in a different fashion. The conductive film is constructed of the flakes overlapping each other. Fig. 1(D) shows that the surface is relatively smooth without observing the overlapping layers of graphite flakes. Under $50 \%$ strain, the graphite flakes separate from the layers below partly to accommodate the deformation (Fig. 1(E)), and after removal of strain fall back into their original positions similar to the previous reports. ${ }^{32}$ The new surface shows ridges where the flakes separated out, Fig. 1(F). The loosely stacked GrMFs slid over each other when under strain, and returned to their initial positions upon stress relaxation. This is analogous to the naturally existing biological scales of animals (e.g. reptiles or fish) which slide over each other when the animal is navigating complex turns and motions. This is why GrMFs are ideal for maintaining electrical contact while undergoing any kind of deformation.

The GrMF/CuNW/GrMF percolation network was prepared by layer-by-layer coating, which is in essence the two aforementioned networks in a sandwich form. The hybrid coating, Fig. 1(G), has a topology similar to Fig. 1(D) due to the GrMF coating being the final layer in both cases. The cracking behaviour after the application of $50 \%$ strain is similar to the GrMF film as well, but in the crevices formed by the sliding graphite layers we can see the CuNW layer acting as a percolation network bridge, Fig. 1(H). Upon relaxation of the strain the cracks close up, much like the earlier observed coatings. So when the GrMFs slide over each other like the scales on reptilian skin and expose a gap, the CuNWs bridge the gap taking advantage of their long length.

The unsealed samples in Fig. 1 exhibit cracking and tearing but this behaviour is diminished to a very large extent when the samples are sealed with a commercial transparent adhesive rubber strip. For instance, the stretchability range of the linear CuNW sensor could be extended from $\sim 50 \%$ to $\sim 280 \%$ after rubber sealing. However, the electrical signals still degraded 
A)

Before

B)

During

C)

After

1
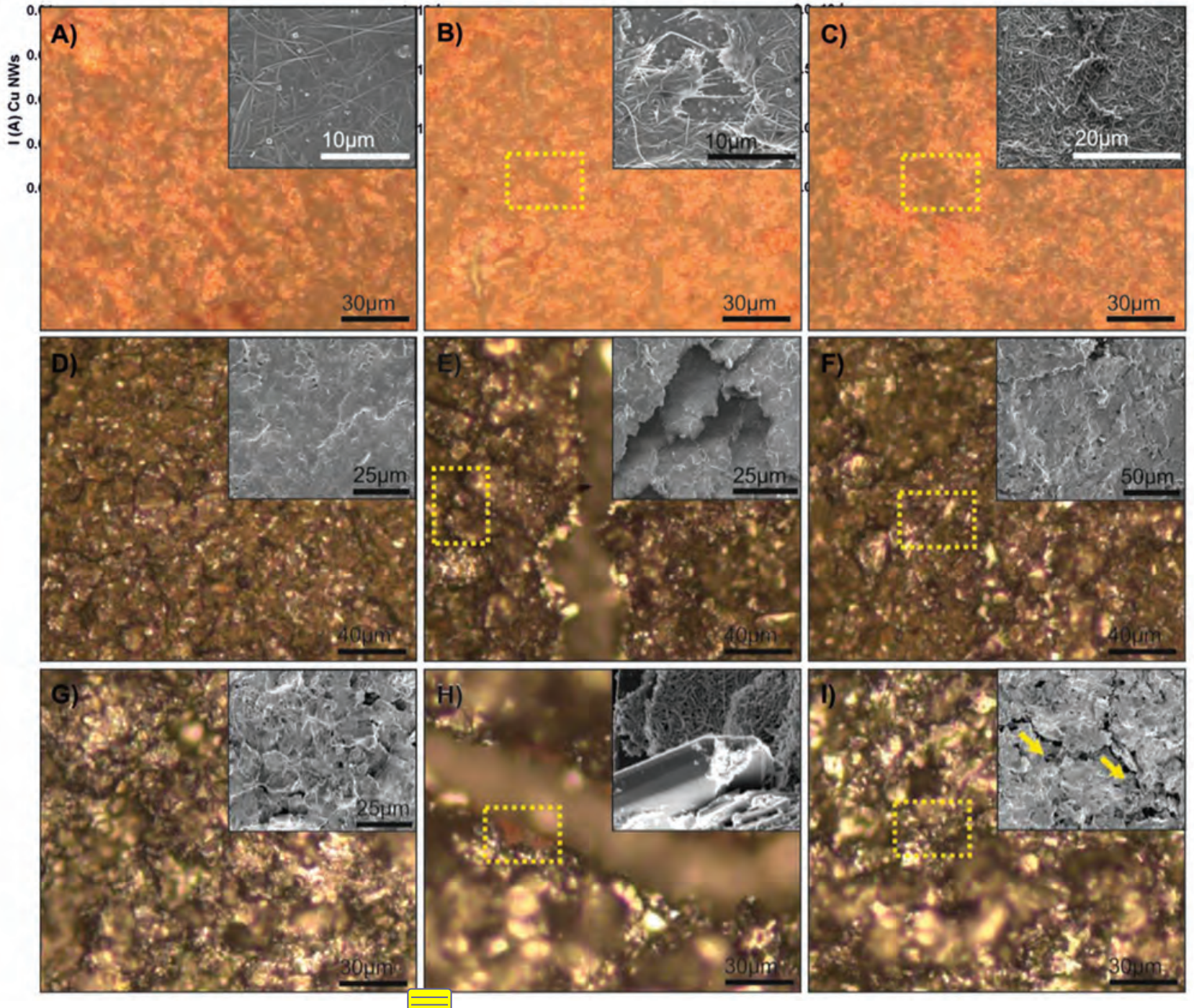

Fig. 1 Optical images of (A) CuNWs, (D) GrMFs, (G) CuNWs/GrMFs, before strain. Images (B, E, H) are during 50\% strain respectively, and (C, F, I) after strain respectively. The inset SEM images show the magnified view of the surface texture, and the material distribution visible through the cracks during strain and after removal of strain.

during the 10100 cycles durability test at $10 \%$ strain as shown in Fig. 2(A). As for GrMFs, Fig. 2(B), sudden spikes and irregular peaks were observed now and then, although generally remaining more stable than the CuNW coating. In contrast, the hybrid film with sealing, Fig. 2(C), clearly shows the clean signal from the CuNWs combined with the stability of the GrMF network. The signal deterioration observed for the CuNWs was also greatly diminished with the hybrid coating. This demonstrated that sealing greatly enhances the strain range, whereas a hybrid conductive coating improves the signal quality by taking advantage of the properties of the individual coatings.

To determine the best material or material combination to detect accurately quick, sharp or minute movements, the sensors with five different conductive coatings, CuNWs, GrMFs, GrMFs/CuNWs/GrMFs, Gr2MFs/CuNWs/
GrMFs, GrMFs/Cu2NWs/GrMFs, were stretched at varying frequencies and the average current change for each coating was plotted. The number "2" after GrMFs and CuNWs signifies two coatings. The sensors were stretched at 1.5, 2.5 and $3 \mathrm{~Hz}$ at $1 \%$ strain for 100 cycles. It is obvious from Fig. 3(A-C) that irrespective of the frequency the GrMF coating has the highest sensitivity, whereas the CuNWs have the lowest. The GrMF/ CuNW/GrMF coatings perform only marginally better than the CuNW coating, while the rest of the coatings perform slightly better than the previous hybrid coating owing to the increased GrMF and CuNW coatings.

As the CuNW coating and the GrMF coating have distinct curve shapes during the cycling tests, it is possible to determine the material playing the dominant role in sensing strain. The visual representation of the dominant materials in a hybrid coating is shown in Fig. S2.† In the CuNW coating 
A)

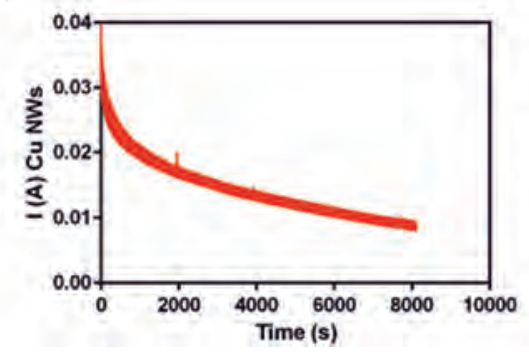

B)

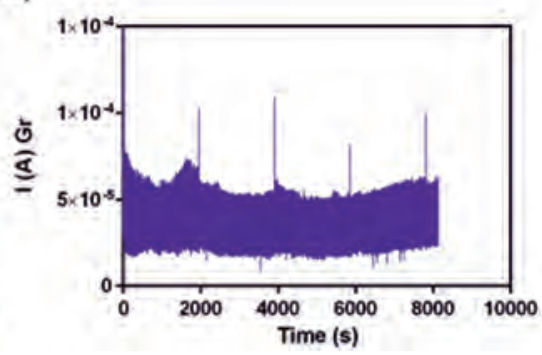

C)

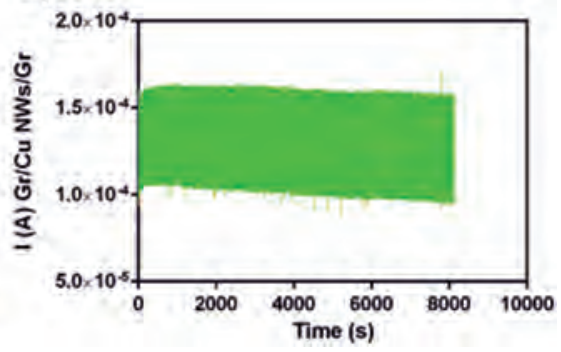

1

Fig. 2 The 10000 cycles test at 10\% strain demonstrating the durability of the coatings, and making a comparison between coatings in terms of signal deterioration for (A) CuNWs, (B) GrMFs, and (C) GrMFs/CuNWs/GrMFs.
A)

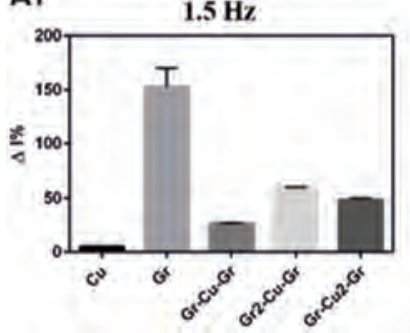

B)

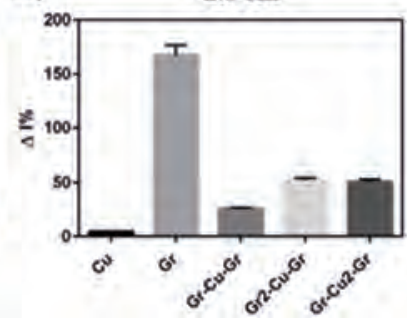

C)

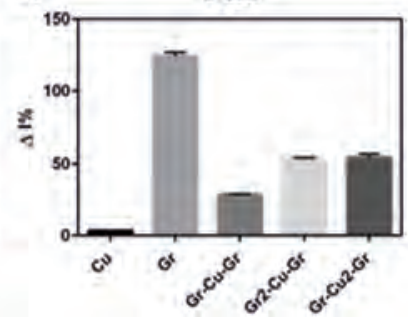

D)

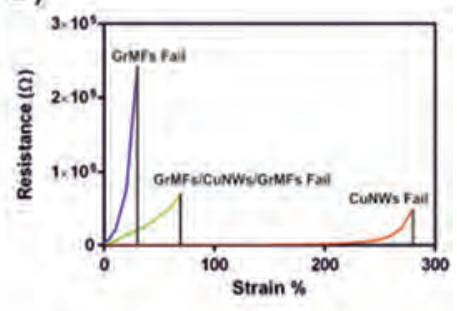

Fig. 3 (A, B, C) Histograms comparing the sensitivities of different coatings (for the linear shape) and showing the influence of varying frequencies. This is to determine the most sensitive coating to detect sharp and minute movements. (D) The failure strain limit of the various conductive coatings.

(Fig. S2(a)†) we can see two broad peaks with two small peaks together in the valley region at the beginning and the middle of the curve at $1.5 \mathrm{~Hz}$. At $2.5 \mathrm{~Hz}$ one of the valley peaks has vanished, and at $3 \mathrm{~Hz}$ all the peaks become defined and regular. The valley peaks may be attributed to the sharp movements that the linear translation stage motor generates when changing the direction for the to and fro motion, and the tall peaks are the changes reflected for the strain. For the GrMF coating, we see pointier peaks reflecting the higher sensitivity of the coating as shown in Fig. S2(b). $\dagger$ The twin valley peaks in this case survive the $2.5 \mathrm{~Hz}$ frequency but one of them is lost at $3 \mathrm{~Hz}$. The curve in Fig. S2(c) $\dagger$ for the GrMF/CuNW/GrMF coating looks like the averaged out value embodying characteristics of both CuNW and GrMF coatings. The hybrid curve contains all the peaks that exist at the particular frequency for both the individual component coatings. With an extra coating of GrMFs the hybrid curve in Fig. S2(d) † has more similarity to Fig. S2(b), $\dagger$ whereas with an extra CuNW coating Fig. S2(e) $\dagger$ looks no different from Fig. S2(c). $\dagger$ With the increase in frequency the jerks are much less pronounced, therefore the loss of peaks may not necessarily reflect the loss of sensitivity.

To reveal how the conductive coating influences the strain range of a sensor it is necessary to find the failure strain limit of the particular coating. The three conductive inks were painted on a linear sensor and stretched to failure i.e. infinite resistance reading. The GrMFs had a limit of $30 \%$, the hybrid coating $70 \%$ and the CuNWs $280 \%$, Fig. 3(D). It is obvious that the extraordinary strain range is the strength of the CuNW coating as proven in Fig. 3(A-C), while the extremely high sensitivity is the strength of the GrMFs, as also seen in Fig. S3. $\dagger$ The strain range of the hybrid coatings is found to be a compromise between the former individual coatings.

It is expected that wrinkles on the skin will influence its sensitivity and stretchability to external strains. To understand this, we fabricated sensors with a few basic first order fractal shapes, namely linear, zigzag, serpentine, and square shape. These shaped sensors were supported on latex substrates as shown in Fig. 4(A-C). Then the CuNW ink was painted and dried on the substrates at room temperature, and finally sealed with a commercial transparent adhesive rubber tape. The $\alpha(\mathrm{s})$ of the shapes were measured to be linear, 6.6; zigzag, 8.3; serpentine, 10.6; square, 14.3. The various shapes have different $\alpha(\mathrm{s})$, and therefore the Poisson effect affects them differently as well. The shrinkage in width of the latex substrate due to transversal compression during strain occurs in the following order: linear > zigzag > serpentine > square. As is evident from the images the linear shape is affected the most, followed by the zigzag shape, and as the square shape is stiffer than the serpentine shape because of its angularity it is more susceptible than the serpentine shape. It should also be noted that the shapes that undergo out-of-axis displacement, square and serpentine, are more compliant to strain than the other shapes. Theoretically, the more angles a shape have the more tensile stiffness it will have. Hence, the descending order of tensile stiffness for the fractal shapes are square > zigzag $>$ serpentine, with the linear shape being most stiff due to its non-fractalness. 
A)

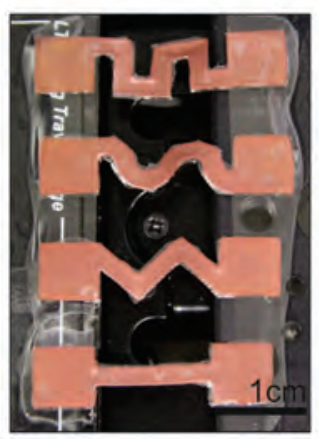

B)

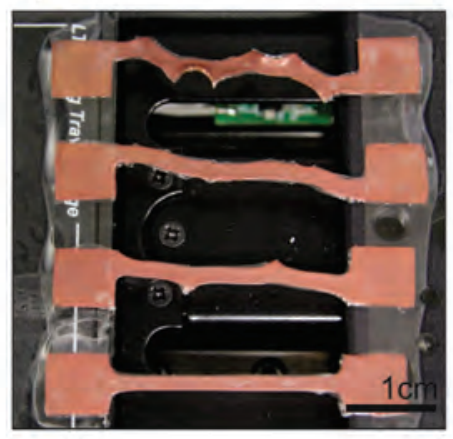

C)

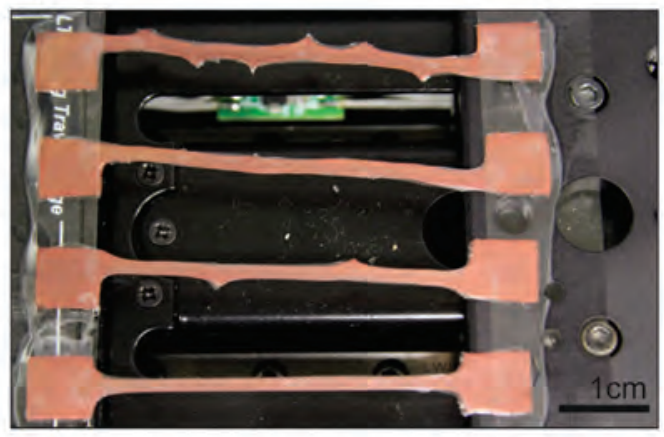

F) Gr MFs - Cu NWs - Gr MFs

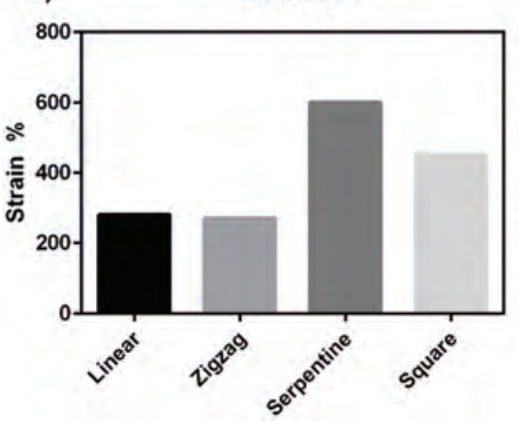

E)

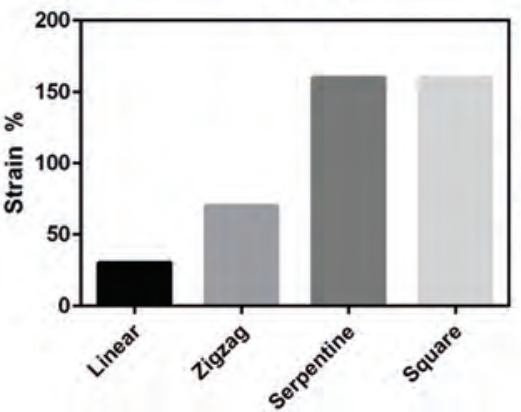

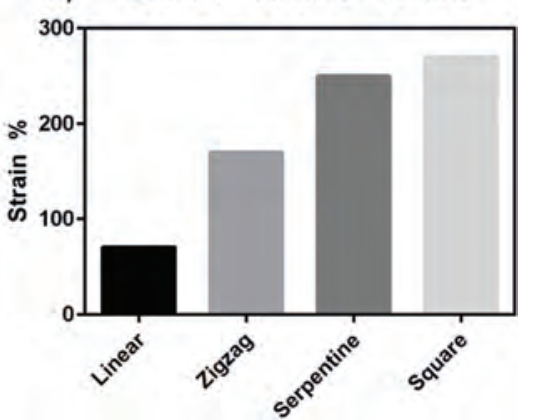

15

20

Fig. 4 The fractal shapes linear, zigzag, serpentine and square being painted with CuNWs at (A) 0\% strain, (B) 100\% strain, and (C) at 200\% strain respectively. The strain range of the different shapes influenced by the different conductive paints: (D) CuNWs, (E) GrMFs, and (F) GrMFs/CuNWs/ GrMFs.
Another aspect to be noted here is that the tensile stiffness may be reduced by increasing the number of bends in the fractal i.e. using a higher order fractal. ${ }^{21,22,33}$ In relation to the conductive coatings, a high tensile stiff shape will result in a higher sensitivity but a lower strain range and vice versa. This is due to the less tolerance for strain by the shape and early failure of the conductive coating. The linear sensor with the smallest $\alpha$ endures the maximum effect of the Poisson effect which causes the conductive coating to be greatly affected. This causes the sensitivity to be the highest in the below $10 \%$ strain ranges, and the ultimate strain to be the least of all shapes. In contrast, the square shaped sensor which has the largest $\alpha$ absorbs much of the strain through out of plane twisting. But owing to its angularity it is rendered stiffer, hence it shows the second largest strain range at almost $450 \%$ instead of the serpentine shape which despite having a lower $\alpha=10.6$ has a higher strain range at $600 \%$. The zigzag shape has a low $\alpha=8.3$ and is also angular, hence it is ranked after the linear shape in terms of stretchability.

Apart from shape, the conductive coating used also decides the strain range and the sensitivity for a strain sensor. Therefore the sensors' sensitivities within a strain range with respect to the shapes and coatings have been thoroughly investigated. As is evident in the strain range comparison histograms between different fractal shapes for the three coatings (Fig. 4(D-F)) CuNW coatings display the highest strain ranges; $600 \%$ for the serpentine shape, but they have the lowest sensi- tivities among the shapes. Whereas, the GrMF coatings have the lowest strain ranges; only $30 \%$ for the linear shape, but the highest sensitivities. The sandwich percolation networks of the GrMF/CuNW/GrMF coatings show averaged out characteristics between CuNWs and GrMFs. The corresponding curves showing the ultimate strains at which the sensors fail are depicted in Fig. S3. $\uparrow$ The hybrid coatings' strain limit always lies within the limits of the other two coatings. Fig. S4† also displays this trend of increasing strain range in terms of shapes and conductive coatings.

The effect of tensile stiffness of the wave shapes used was confirmed for two dimensional patterns as well. Rows of the zigzag, the square and the serpentine wave shapes were laid orthogonally to each other to construct second order fractal patterns, as shown in Fig. 5(A-C). The insets show the respective first and second order patterns. These patterns allow for biaxial stretching and torsion. Fig. 5(D) shows that there is a large difference between the resistance changes for strain along the " $X$ "- $\varepsilon(11)$ and " $Y$ " $-\varepsilon(22)$ axes for the zigzag shape. The difference is much less in comparison to the square shape, and the least, almost overlapping for the serpentine shape. It is also observed in the torsion $-\varepsilon(12)$ test, Fig. $5(\mathrm{E})$, that the peak value for resistance changes is the highest for the zigzag shape, followed by square and serpentine. This shows that according to the trend of the Poisson effect influenced by fractalness of the shapes zigzag $>$ square $>$ serpentine, the more fractal a shape is, the less it will be directly affected by biaxial 
A)

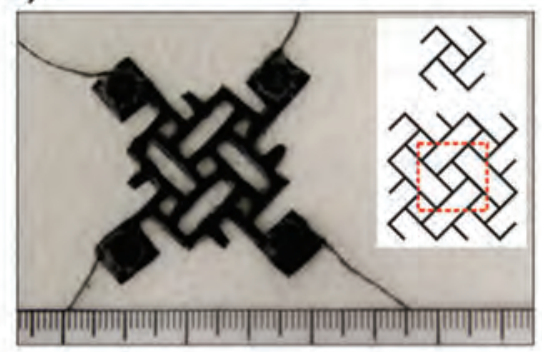

B)

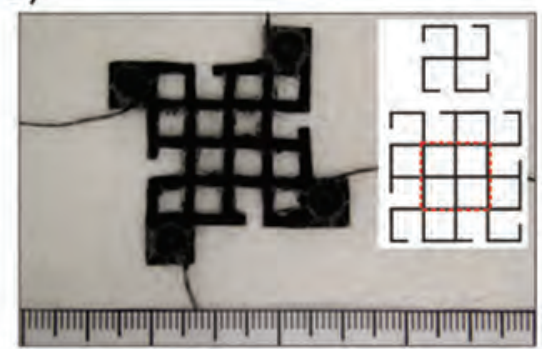

C)

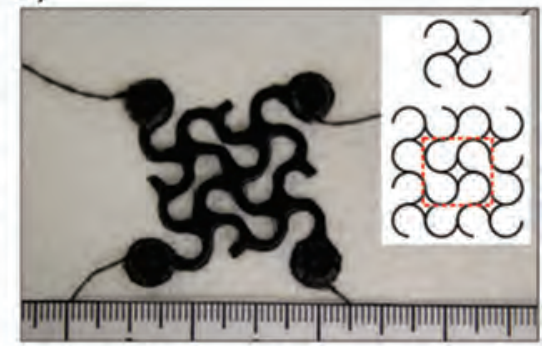

1

5

E)

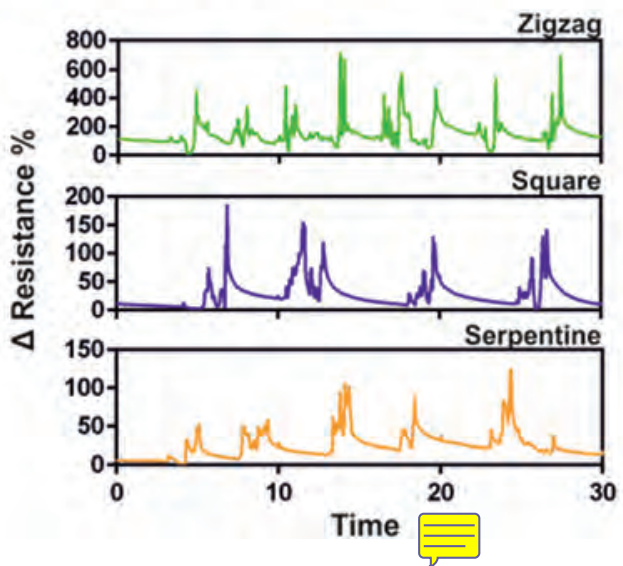

Fig. 5 The second order fractal pattern biaxial and torsion GrMF sensors with the first and second order patterns in the inset schematics: (A) zigzag, (B) square, and (C) serpentine. (D) Resistance changes due to strain in the " $X$ " $-\varepsilon(11)$ and " $Y$ " $-\varepsilon(22)$ axes. The green triangles are $x$-zigzag, purple triangles are $y$-zigzag; blue circles are $x$-square, red squares are $y$-square; orange diamonds are $x$-serpentine and black circles are $y$-serpentine. (E) shows the response for random twists, $\varepsilon(12)$ of the sensors.

strain and torsion. The majority of the applied strain will be spent in uncoiling the fractal, like a spring.

For biomedical monitoring, strain based plethysmographic changes in the human skin over the areas of interest in the human body are measured, such as the skin over arteries, muscles, joints and so on. These kinds of measurements require the sensitivity to be high in the respective strain ranges. For instance, a blood pulse will typically generate an under $1 \%$ strain change, therefore either the GrMFs or the TrMF/CuNW/GrMF linear sensors have a GF 36. Similarly, for measuring proprioceptive musculoskeletal movements the zigzag wave shape sensor having a GF $\sim 6$ for the GrMF coating or GF $\sim 11$ for GrMFs/CuNWs/GrMFs within the $20-60 \%$ strain range would be suitable. For strains in the vicinity of $100 \%$ both coatings except for the CuNW coating show a GF $\sim 100$ regardless of the shape of the sensors. Beyond $100 \%$ strain the sensors reach a sensitivity upwards of GF $\sim 1000$. The amount of detailed minute movements that can be detected by a particular combination of a fractal shape and conductive coating is demonstrated in Fig. S5.† In general the zigzag, serpentine and square are the most sensitive in picking up movements. But when they are coated with CuNWs, all of them show low sensitivity, and look more or less the same. When the shapes are coated with the hybrid coating it was observed that the signal becomes cleaner and more regular. This was especially evident in the linear shape. This demon- strates that it is possible to tailor the sensor to have its highest sensible region in the strain range we desire by using a suitable fractal shape and conductive coating.

We further demonstrate that a coating of CuNWs, GrMFs and a combination thereof could adhere on an ecoflex replica of the human skin with high fidelity. Fig. 6(A) shows the ecoflex skin coated with CuNWs, Fig. 6(C) with GrMFs and Fig. 6(E) shows GrMFs with a coating of CuNWs over it, and Fig. 6(B, D and F) depict the respective magnifications. It should be noted that for the experiments and measurements a sandwich of GrMFs/CuNWs/GrMFs was used. Fig. 6(E) doesn't include the third layer of GrMFs to enunciate visually the placement of the CuNWs on the GrMF layer underneath, and during experiments between the layers of GrMFs. It can be observed that the nanomaterials drape over the many wrinkles and crevices on the ecoflex skin. This shows how the conductive paint is able to accurately replicate the contours of the skin. Fig. 6(D) shows how the GrMFs bind tightly to each other, almost merging into each other. It is also interesting to note that when the graphite is made into an aqueous paint, it is only then that the microflakes settle into a tightly bound snake-skin scale like arrangement. The water seems to have an intercalating effect at the micron level if not at the nano level and separates out multilayered graphene flakes. The same intercalating effect doesn't happen on rubbing the graphite on a surface as done with a pencil, as shown in Fig. S6.† 

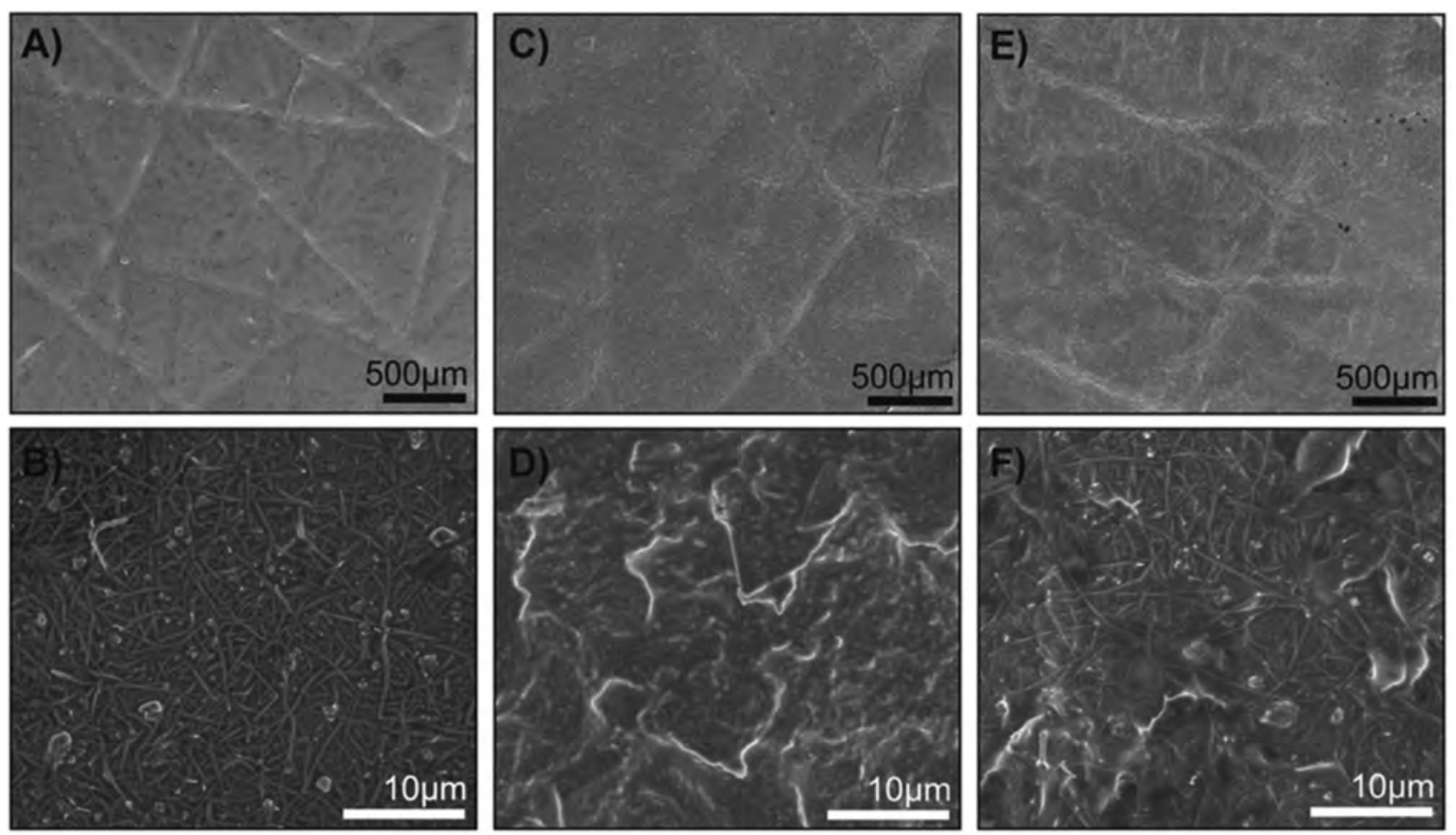

Fig. 6 The conductive paints shown to be intimately contacting the many wrinkles and folds on an ecoflex replica of the human skin. (A) CuNWs, (C) GrMFs, and (E) CuNWs-GrMFs and the respective magnifications in (B, D, F).

As demonstrated above, our 'paint-on' approach could potentially lead to personalised wearable sensors conformal to local skin textures with adjustable sensitivities and stretchabilities. This displays the potential in monitoring a wide range of biometric signals. The GrMFs were painted as a single patch directly on the inner wrist and then sealed with a transparent adhesive rubber strip, Fig. S7(a), $\dagger$ and this sensor was used to detect skin strains caused by the movement of the tendons underneath. The sensor is able to detect each finger movement distinctly, followed by palm flexion and extension, Fig. 7(A). Each movement was made twice to show reproducibility. This could be potentially used as a prosthetic interface for hand amputees. The GrMFs were also painted onto the posterior tibial artery site to detect the ankle brachial pulse, Fig. 7(B), like a tattoo in the shape of a bat, Fig. $S 7(b) . \dagger$ The sensor could very easily pick up the early systolic (p1), late systolic (p2) pressure peaks, and even the dicrotic notch (p3) in the pulse wave, as shown in the inset. The demonstration can be viewed in ESI Video S1. $\dagger$ The ability to avoid intrusive catheter based methods or
A)

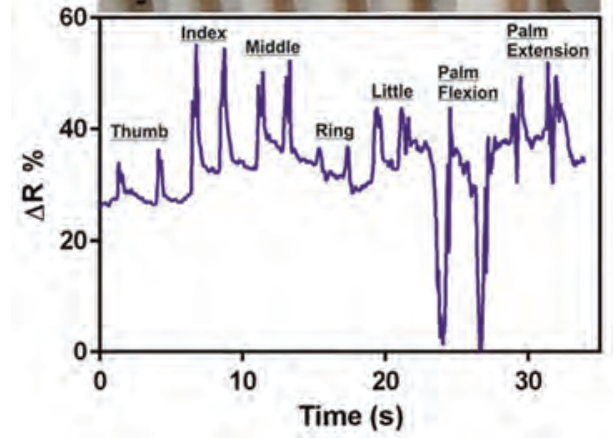

B)

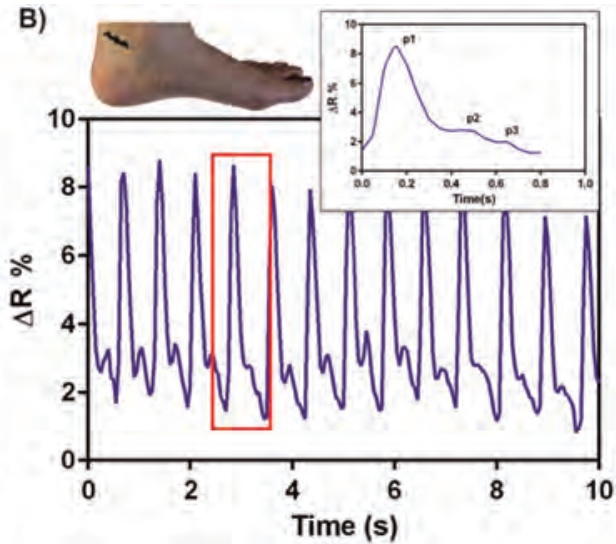

Fig. 7 (A) Demonstration of a single GrMF sensor painted directly on the inner wrist used to detect and differentiate between individual finger motions and hand movements. (B) GrMF ink painted on the posterior tibial artery site in the shape of a bat shaped tattoo to detect ankle brachial artery pulse. The inset shows the pulse wave. 
1 uncomfortable pressure cuff based methods in favour of a facile paintable pulse sensor has huge implications in the medical field. Ideally, for measuring the pulse wave velocity the carotid artery on the neck and any other artery site on the body's extremities would give a very accurate read out.
But due to the discomfort of having a pressure cuff on the neck, the arms and the legs are chosen as the measurement sites. Now, the availability of a non-intrusive, imperceptible paintable strain sensor allows for applying sensors on any artery site on the body with no discomfort.

A)

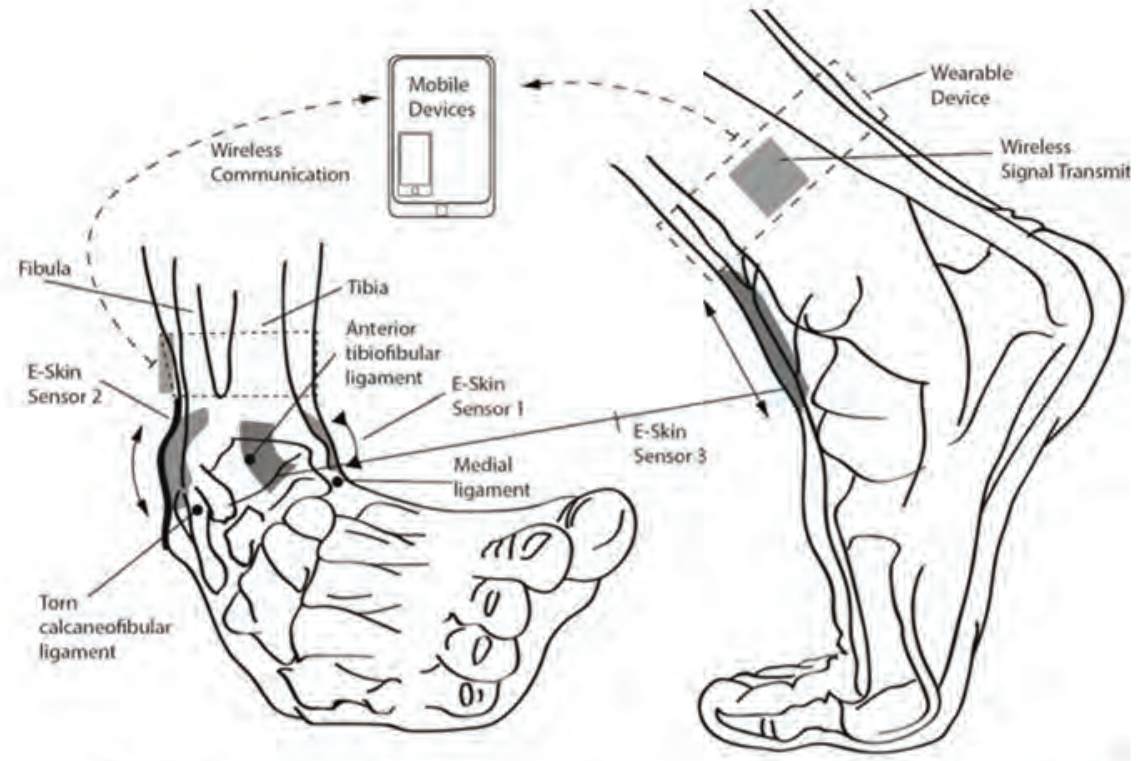

B)
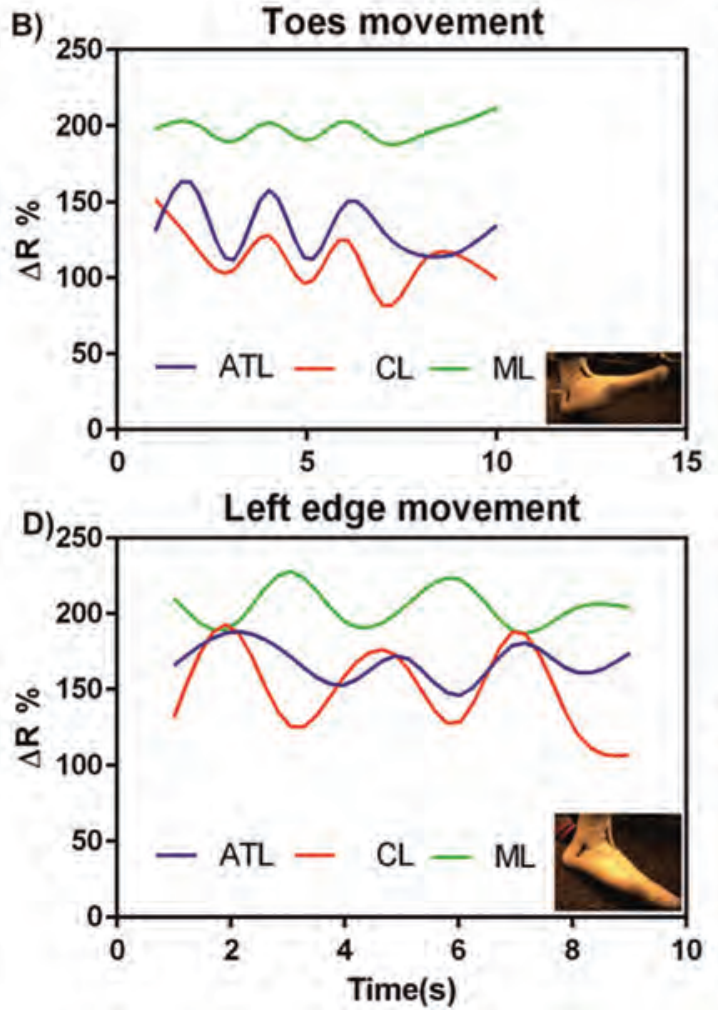

C)

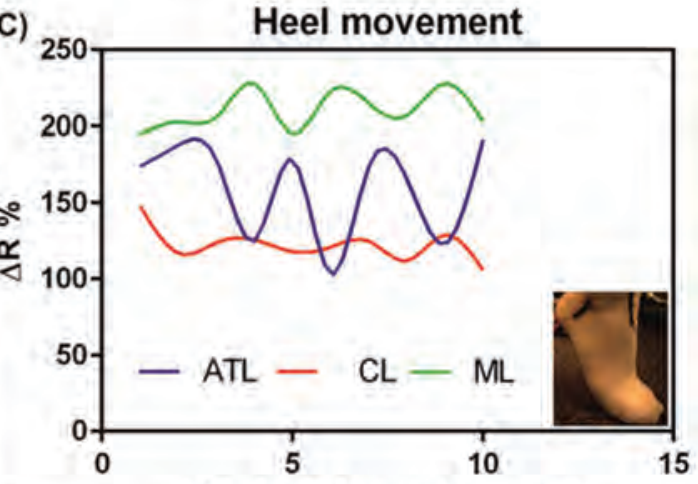

E)

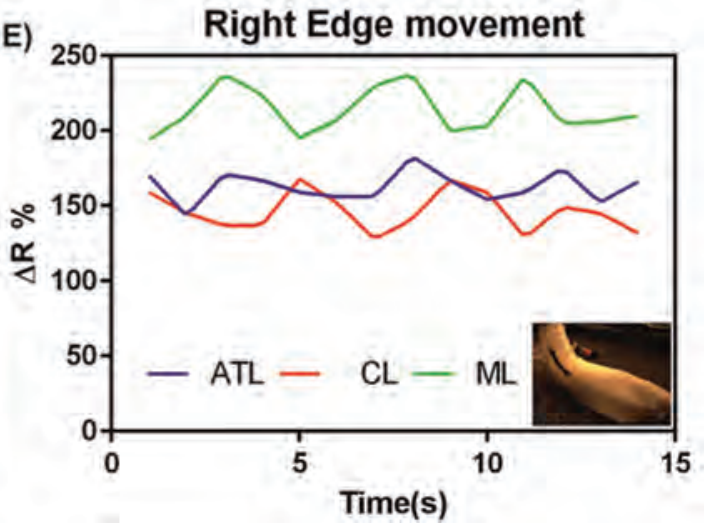

1

Fig. 8 (A) Schematic depicting the placement of the CuNW/GrMF/CuNW sensors on the ankle skin locations corresponding to the anterior tibiofibular ligament (ATL), calcaneofibular ligament (CL), and medial ligament (ML). The wireless device placed on the sensory sock can communicate with smart devices and deliver data in real time. Ankle motion sensors with the wireless platform demonstrating, (A) toes and (B) heel up-down movement, and side-to-side hinge movement on the (C) left and (D) right foot edge, and their corresponding resistance change readouts from the ankle sensor set. 
The GrMF/CuNW/GrMF paint was painted on a sock and positioned such that the skin strains caused by the calcaneofibular, medial, and anterior-tibiofibular ligaments respectively could be measured. The schematic, Fig. 8(A), shows how the three sensors are connected to a wireless platform which can communicate with a mobile device and show real time data while the ankle is in motion. This sock is an ideal wearable sensor platform designed to monitor ankle movement during sporting actions in real time. The inset images illustrate what kind of position of the ankle would risk causing a torn ligament. Fig. 8(B-E) depict the raw data collected from the ankle for the respective foot motions. Each movement was made three times to demonstrate the consistency of the measurements. Each set of waveforms is distinct for each movement. This setup is ideal for sporting, physiotherapy and rehabilitation based applications. ${ }^{34}$ This is aimed at injury prevention by accurate proprioceptive knowledge of the ankle during motion. The demonstration of this sensor can be viewed in ESI Videos S2 and S3. $\dagger$

\section{Conclusions}

In conclusion, this work represents a thorough study of various conductive coatings CuNWs, GrMFs and the combinations thereof, on the latex substrates and their behaviour under strain. The different shaped latex substrates: linear, zigzag, serpentine, and square, add another layer of tunability by introducing aspect ratio and Poisson effect based deformations and their effect on the conductive coatings. Using the facile "paint-on" approach it is possible to develop e-skin sensors which can directly be fabricated on the human skin hence giving us a high fidelity, and user specific skin replica of the surface topology. This enables us to have high accuracy for biometric data collection. Within the $10 \%$ range a very high GF $\sim 36$ and a maximum GF $\sim 3000$ overall were displayed by the sensors, which is well suited for a plethora of applications including biomonitoring, prosthetic and sporting applications. The extremely high stretchability of $600 \%$ again offers applications in the robotics area. All the sensors have displayed a durability of 10000 cycles at $10 \%$ strain, Fig. S8. $\dagger$ The facile fabrication route, robustness, and economically viable nature of the "paintable" sensors make them ideal for healthcare needs in developing nations. The "paintable" and "wireless" nature of the sensors allows for a cuff free measurement and collection of blood pulse and plethysmography data. This is especially advantageous for infants and geriatric patients. The GrMF based sensors may be immediately deployed for this cause.

\section{Experimental section}

\section{Materials}

Hydroxypropyl cellulose (HPC, Sigma-Aldrich, 20 mesh, $M_{\mathrm{w}}=$ 1000 000), hexadecylamine (HDA, Sigma-Aldrich), D-glucose
( $\alpha$ or $\beta$, Merck), copper chloride $\left(\mathrm{CuCl}_{2} \cdot 2 \mathrm{H}_{2} \mathrm{O}\right.$, Sigma-Aldrich), 1 and LYRA water soluble graphite crayons $9 \mathrm{~B}$.

\section{CuNW synthesis}

The CuNWs were synthesized using a modified earlier published method. ${ }^{35} 50 \mathrm{~mL}$ of water was heated to $100{ }^{\circ} \mathrm{C}$. Then $900 \mathrm{mg}$ of hexadecylamine (HDA) and $100 \mathrm{mg}$ of copper chloride $\left(\mathrm{CuCl}_{2} \cdot 2 \mathrm{H}_{2} \mathrm{O}\right)$ were added and stirred for $20 \mathrm{~min}$ at $1000 \mathrm{rpm}$. When the solution turns into a homogeneous sky blue color, $500 \mathrm{mg}$ of D-glucose ( $\alpha$ or $\beta$ ) (Merck) was added and the stirring speed was reduced to $400 \mathrm{rpm}$. The solution gradually changed colour from pale brown to dark brown. The reaction was stopped after 6 hours. The solution was removed from the oil bath and was cooled at room temperature for $10 \mathrm{~min}$ after which it was centrifuged at $6500 \mathrm{rpm}$ for $5 \mathrm{~min}$. The CuNWs collect at the bottom of the tubes as a pellet, which was recovered by carefully decanting the supernatant and gently rinsing with Milli-Q water a few times. The CuNWs formed are 25-35 $\mathrm{nm}$ in diameter and 50-60 $\mu \mathrm{m}$ long.

\section{CuNW ink formulation}

$1 \mathrm{~g}$ (wet pellet weight) of CuNWs, $9.468 \mathrm{mg}$ of HPC, and $2.2 \mathrm{ml}$ of Milli-Q water were blended well to make the ink.

\section{GrMF ink formulation}

$300 \mathrm{mg}$ of shavings from a water soluble graphite crayon were dissolved in $20 \mu \mathrm{L}$ of Milli-Q water to make the ink. The GrMFs are $<10 \mu \mathrm{m}$ in diameter and the thickness can be anywhere between a few $100 \mathrm{~nm}$ to $1 \mu \mathrm{m}$ depending on the number of layers.

\section{Characterization}

High-resolution images of the CuNWs were captured using an FEI Nova NanoSEM 450 FESEM.

\section{Sensor fabrication}

The elastomer substrates are prepared by pouring the liquid latex into $3 \mathrm{D}$ printed moulds and then curing at $70{ }^{\circ} \mathrm{C}$ for 30 minutes. The conductive threads were stuck on the ends of the sensors as electrodes using the liquid latex. The required conductive paint was painted on the top surface of the sensor using a paintbrush. After drying the assembly, the dried paint was sealed on the sensor using commercial transparent stretchable medical rubber tape, OPSITE FLEXIFIX.

\section{Acknowledgements}

The research has been funded by the Australian Research Council DP15013750. This work was performed in part at the Melbourne Centre for Nanofabrication (MCN) in the Victorian Node of the Australian National Fabrication Facility (ANFF). The authors acknowledge the use of the microscopy facilities within the Monash Centre for Electron Microscopy (MCEM). The author NNJ also wishes to acknowledge the Victorian International Research Scholarship (VIRS) for their support. 
$1 \quad$ References

1 V. Nayyar, K. Ravi-Chandar and R. Huang, Int. J. Solids Struct., 2011, 48, 3471.

52 V. Arumugam, M. D. Naresh and R. Sanjeevi, J. Biosci., 1994, 19, 307.

S. Gong, D. T. H. Lai, B. Su, K. J. Si, Z. Ma, L. W. Yap,

P. Guo and W. Cheng, Adv. Electron. Mater., 2015, 1.

4 S. Gong, D. T. Lai, Y. Wang, L. W. Yap, K. J. Si, Q. Shi, N. N. Jason, T. Sridhar, H. Uddin and W. Cheng, ACS Appl. Mater. Interfaces, 2015, 7, 19700.

5 L. Cai, L. Song, P. Luan, Q. Zhang, N. Zhang, Q. Gao, D. Zhao, X. Zhang, M. Tu, F. Yang, W. Zhou, Q. Fan, J. Luo, W. Zhou, P. M. Ajayan and S. Xie, Sci. Rep., 2013, 3, 3048.

6 S.-H. Bae, Y. Lee, B. K. Sharma, H.-J. Lee, J.-H. Kim and J.-H. Ahn, Carbon, 2013, 51, 236.

7 Y. Wang, T. Yang, J. Lao, R. Zhang, Y. Zhang, M. Zhu, X. Li, X. Zang, K. Wang, W. Yu, H. Jin, L. Wang and H. Zhu, Nano Res., 2015, 8, 1627.

8 M. Amjadi, A. Pichitpajongkit, S. Lee, S. Ryu and I. Park, ACS Nano, 2014, 8, 5154.

9 N. N. Jason, W. Shen and W. Cheng, ACS Appl. Mater. Interfaces, 2015, 7, 16760.

10 X. Li, R. Zhang, W. Yu, K. Wang, J. Wei, D. Wu, A. Cao, Z. Li, Y. Cheng, Q. Zheng, R. S. Ruoff and H. Zhu, Sci. Rep., 2012, 2, 870.

11 J. Song and H. Zeng, Angew. Chem., Int. Ed., 2015, 54, 9760.

12 J. Song, J. Li, J. Xu and H. Zeng, Nano Lett., 2014, 14, 6298.

13 G. Schwartz, B. C. Tee, J. Mei, A. L. Appleton, H. Kim do, H. Wang and Z. Bao, Nat. Commun., 2013, 4, 1859.

14 Y. Wang, L. Wang, T. Yang, X. Li, X. Zang, M. Zhu, K. Wang, D. Wu and H. Zhu, Adv. Funct. Mater., 2014, 24, 4666.

15 T. Yamada, Y. Hayamizu, Y. Yamamoto, Y. Yomogida, A. Izadi-Najafabadi, D. N. Futaba and K. Hata, Nat. Nanotechnol., 2011, 6, 296.

16 S. Gong, W. Schwalb, Y. Wang, Y. Chen, Y. Tang, J. Si, B. Shirinzadeh and W. Cheng, Nat. Commun., 2014, 5, 3132.

17 C. Pang, G. Y. Lee, T. I. Kim, S. M. Kim, H. N. Kim, S. H. Ahn and K. Y. Suh, Nat. Mater., 2012, 11, 795.

18 S. Lim, D. Son, J. Kim, Y. B. Lee, J.-K. Song, S. Choi, D. J. Lee, J. H. Kim, M. Lee, T. Hyeon and D.-H. Kim, Adv. Funct. Mater., 2015, 25, 375.
19 W. H. Yeo, Y. S. Kim, J. Lee, A. Ameen, L. Shi, M. Li, S. Wang, R. Ma, S. H. Jin, Z. Kang, Y. Huang and J. A. Rogers, Adv. Mater., 2013, 25, 2773.

20 R. C. Webb, A. P. Bonifas, A. Behnaz, Y. Zhang, K. J. Yu, H. Cheng, M. Shi, Z. Bian, Z. Liu, Y. S. Kim, W. H. Yeo, J. S. Park, J. Song, Y. Li, Y. Huang, A. M. Gorbach and J. A. Rogers, Nat. Mater., 2013, 12, 938.

21 S. Xu, Y. Zhang, J. Cho, J. Lee, X. Huang, L. Jia, J. A. Fan, Y. Su, J. Su, H. Zhang, H. Cheng, B. Lu, C. Yu, C. Chuang, T. I. Kim, T. Song, K. Shigeta, S. Kang, C. Dagdeviren, I. Petrov, P. V. Braun, Y. Huang, U. Paik and J. A. Rogers, Nat. Commun., 2013, 4, 1543.

22 J. A. Fan, W. H. Yeo, Y. Su, Y. Hattori, W. Lee, S. Y. Jung, Y. Zhang, Z. Liu, H. Cheng, L. Falgout, M. Bajema, T. Coleman, D. Gregoire, R. J. Larsen, Y. Huang and J. A. Rogers, Nat. Commun., 2014, 5, 3266.

23 D.-H. Kim, R. Ghaffari, N. Lu, S. Wang, S. P. Lee and H. Keum, Proc. Natl. Acad. Sci. U. S. A., 2012, 109, 19910.

24 S. Yang, Y. C. Chen, L. Nicolini, P. Pasupathy, J. Sacks, 二. Becky, R. Yang, S. Daniel, Y. F. Chang, P. Wang, D. Schnyer, D. Neikirk and N. Lu, Adv. Mater., 2015.

25 J. T. Muth, D. M. Vogt, R. L. Truby, Y. Menguc, D. B. Kolesky, R. J. Wood and J. A. Lewis, Adv. Mater., 2014, 26, 6307.

26 A. R. Rathmell, M. Nguyen, M. Chi and B. J. Wiley, Nano Lett., 2012, 12, 3193.

27 I. E. Stewart, A. R. Rathmell, L. Yan, S. Ye, P. F. Flowers, W. You and B. J. Wiley, Nanoscale, 2014, 6, 5980.

28 A. Clausen, F. Wang, J. S. Jensen, O. Sigmund and J. A. Lewis, Adv. Mater., 2015, 27, 5523.

29 M. Park, J. Im, M. Shin, Y. Min, J. Park, H. Cho, S. Park, M. B. Shim, S. Jeon, D. Y. Chung, J. Bae, J. Park, U. Jeong and K. Kim, Nat. Nanotechnol., 2012, 7, 803.

30 Y. Tang, S. Gong, Y. Chen, L. W. Yap and W. Cheng, ACS Nano, 2014, 8, 5707.

31 Y. Won, A. Kim, W. Yang, S. Jeong and J. Moon, NPG Asia Mater., 2014, 6, e132.

32 M. Hempel, D. Nezich, J. Kong and M. Hofmann, Nano Lett., 2012, 12, 5714.

33 Y. Su, S. Wang, Y. Huang, H. Luan, W. Dong, J. A. Fan, Q. Yang, J. A. Rogers and Y. Huang, Small, 2015, 11, 367.

34 S. J. Wang, Fields Interaction Design (FID): The answer to ubiquitous computing supported environments in the post-information age, Homa \& Sekey Books, 2013.

35 M. Jin, G. He, H. Zhang, J. Zeng, Z. Xie and Y. Xia, Angew. Chem., Int. Ed., 2011, 50, 10560. 